\title{
Analysis of metallic impurity content by means of VUV and SXR diagnostics in hybrid discharges with hot-spots on the JET-ILW poloidal limiter
}

\author{
A. Czarnecka ${ }^{1}$, N. Krawczyk ${ }^{1}$, P. Jacquet ${ }^{2}$, E. Lerche ${ }^{3}$, V. Bobkov ${ }^{4}$, C. Challis ${ }^{2}$, D.
} Frigione $^{5}$, J. Graves ${ }^{6}$, K. D. Lawson², M.J. Mantsinen ${ }^{7,8}$, L. Meneses ${ }^{9}$, E. Pawelec ${ }^{10}$, T. Pütterich4, M. Sertoli $^{4,2}$, M. Valisa ${ }^{11}$, D. Van Eester ${ }^{3}$, and JET Contributors ${ }^{12}$

EUROfusion Consortium, JET, Culham Science Centre, Abingdon, OX14 3DB, UK

${ }^{1}$ Institute of Plasma Physics and Laser Microfusion, Hery 23 Str., 01-497 Warsaw, Poland

${ }^{2}$ CCFE, Culham Science Centre, Abingdon, OX14 3DB, UK

${ }^{3}$ Laboratory for Plasma Physics, ERM/KMS, EUROfusion Consortium Member

Renaissancelaan 30, B-1000 Brussels, Belgium

${ }^{4}$ Max-Planck-Institut für Plasmaphysik, Boltzmannstr.2, D-85748, Germany

${ }^{5}$ ENEA, Fusion and Nuclear Safety Dep., C.R. Frascati, Frascati (Roma), Italy

${ }^{6}$ Ecole Polytechnique Fédérale de Lausanne (EPFL), Swiss Plasma Center (SPC), CH-1015 Lausanne, Switzerland

${ }^{7}$ ICREA, Barcelona, Spain,

${ }^{8}$ Barcelona Supercomputing Center, Barcelona, Spain

${ }^{9}$ Associação EURATOM/IST, Instituto de Plasmas e Fusão Nuclear - Laboratório Associado, Instituto

Superior Técnico, P-1049-001 Lisboa

${ }^{10}$ Institute of Physics, Opole University, Oleska 48 Str., 45-052 Opole, Poland

${ }^{11}$ Consorzio RFX, Padova, Italy

${ }^{12}$ See the author list of "X. Litaudon et al 2017 Nucl. Fusion 57 102001"

E-mail: agata.czarnecka@ifpilm.pl

Received xxxxxx

Accepted for publication $\mathrm{xxxxxx}$

Published xxxxxx

\begin{abstract}
In preparation for the upcoming JET D-T campaign, great effort has been devoted during the 2015-2016 JET campaigns with the ITER-like wall (ILW) to the extension of the high-performance H-mode phase in baseline and hybrid scenarios. Hybrid discharges were the only ones that have been stopped by the real-time vessel protection system due hot-spot formation on the outboard poloidal limiter. Generation of hot-spots was linked to the application of high NBI and ICRH power. In tokamaks with high-Z plasma components, the use of ICRH heating is also accompanied by an increased metallic impurity content. Simultaneous control of hot-spot temperature and the core impurity content was crucial due to the fact that the same plasmawall interaction mechanism is responsible for both phenomena. Impurity data collected by SXR, EUV and VUV diagnostics were able to provide for the first time comprehensive information concerning tungsten and mid-Z impurities such as nickel, iron, and cooper. To determine absolute mid-Z impurity concentrations a new relative calibration technique, compatible with JET-ILW, has been developed based on cross-calibration with a calibrated spectrometer via the quasicontinuum of W in the 200-400 $\AA$ wavelength range. In hybrid discharges, it was found that local $\mathrm{D}_{2}$ gas injection, plasma current, separatrix density, and fast ion losses appeared to impact hot-spot temperature and core impurity levels. Analysis showed a reduced maximum hot-spot temperature and impurity concentration at higher gas rate. Changes in the plasma current had a strong impact on the plasma-wall interaction, both via modifications in the edge density and in the fast-ion losses. At constant gas injection rate, both the hot-spot temperature and the core impurity content decreased with the separatrix density. The main mechanism
\end{abstract}


responsible for the formation of the hot-spots was found to be linked to the fast ion losses, but RF-sheath effects may also be playing a role in the high limiter temperatures observed in these experiments.

Keywords: tokamaks, plasma impurity, tungsten, VUV spectroscopy, hot-spots

\section{Introduction}

Experimental campaigns performed at the tokamak JET in 2015-2016 were devoted to the optimisation of ITER-relevant operating regimes in the presence of the ITER-Like Wall (ILW) and to the optimal preparation of the JET deuteriumtritium (JET-DT) campaign [1-2]. The development of operational scenarios is underway with the target to provide a maximum of about $15 \mathrm{MW}$ of fusion power in the forthcoming JET DT experiment, maintaining steady high performance Hmode phase for $\sim 5$ seconds. This requires i) high power heating, ii) low neutral density (i.e. low gas injection rate and good pumping to minimize power degradation of confinement), iii) balance between high plasma current (for high global confinement), and iv) low plasma density (for central beam deposition and high core temperature, $\mathrm{T}_{\mathrm{i}}>\mathrm{T}_{\mathrm{e}}$ ). The main challenge to achieve such conditions are high $\mathrm{Z}$ impurity accumulation, high divertor heat loads and MHD instabilities. For this purpose, 'baseline' plasmas at high plasma current and, hence, low q95 were offten considered. This plasma scenario takes advantage of the favourable scaling of plasma energy confinement with plasma current to achieve high performance. Also 'hybrid' plasmas at reduced plasma current and, hence, higher $\mathrm{q}_{95}$ were being developed. In this domain higher normalised pressure $\left(\beta_{\mathrm{N}}\right)$ can be achieved. It was found that higher confinement factor $\mathrm{H} 98(\mathrm{y}, 2)$ can be accessed at higher $\beta_{\mathrm{N}}$ [3], which helps to compensate for the reduction in plasma current in terms of energy confinement. Operation at higher $\beta_{\mathrm{N}}$ is challenging in terms of MHD stability. Also, the reduction in plasma current for 'hybrid' plasmas compared with 'baseline' plasmas tends to result in reduced plasma density, which has potential advantages for JET DT because of the improved beam penetration and, hence, central heating. In experiments in 2016, in both baseline and and hybrid scenarios a new ILW fusion record of $2.9 \times 10^{16} \mathrm{DD}$ neutrons/s was achieved [4]. High-Z impurity control in the core of D-T scenarios with Ion Cyclotron Resonance Heating (ICRH) was crucial for extending the duration of the high-performance $\mathrm{H}$-mode phase [4]. In JET-ILW, core impurity screening can be achieved with high power ICRH heating $[5,6]$. However, due to RF sheath rectification effects [7] and in some cases fast ion losses (both potentially causing enhanced plasma-wall interaction), increased edge sources were also observed during the application of high power ICRH. For core impurity control, fundamental $\mathrm{H}$ minority ICRF heating with low minority concentration and on-axis ICRH deposition profile has shown the best results so far in high power H-mode plasmas [5-6,8-
9], but dominant second harmonic deuterium ( $N=2$ D) heating also proved to be effective [11]. $\mathrm{He}^{3}$ and/or combined $\mathrm{H}+\mathrm{He}^{3}$ minority ICRH heating has shown some promising results in a few cases studied [11] but more experimental investigation is needed to establish these scenarios as a workhorse for impurity control in JET-ILW. The main effects of ICRH that cause reduced impurity peaking were related to temperature peaking, density flattening and fast ion effects [8-10] such as fast ion anisotropy and the collisional processes. Therefore, for plasma operation it is important to characterize the impurity behavior in the confined plasma, as impurities may lead to radiative cooling and core localized impurity accumulation. In this paper special focus was given to highpower hybrid discharges, conducted during last campaign at JET. Hybrid discharges were the only ones that have been stopped by the real-time vessel protection system because of outer-wall hot-spot formation, probably as a combination of the specific q95 used in these discharges (much higher than the baseline) and the lower core collisionality which can potentially lead to faster ions, larger orbits and enhanced fast ion losses. The enhanced heat-loads were believed to be related to the joint application of high neutral beams injection (NBI) and ICRH power. Figure 1 shows the image from a visible camera with the indicated high temperature region on the narrow poloidal limiter NPL3B close to the ICRH antenna B. Formation of hot-spots on a first wall surface can result in a strong impurity and dust ejection into plasma and discharge termination.

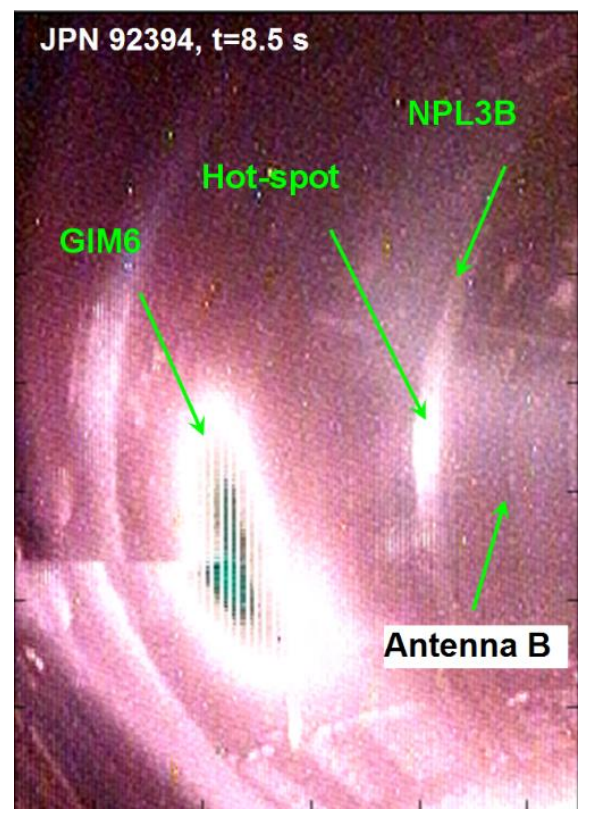


Figure 1. Image from JET visible camera in discharge 92394 at $\mathrm{t}=8.5$ $\mathrm{s}$, with the high temperature regions (hot-spot) in the poloidal limiter NPL3B, close to the ICRH antenna B structure and with the location of the the mid-plane gas injection module (GIM6).

In the performed experiment it was observed that local deuterium $\left(\mathrm{D}_{2}\right)$ gas injection can mitigate hot-spot formation. In connection with the above, strong emphasis was given on the metalic imputity content in the plasma core. Therefore, both influence of $\mathrm{D}_{2}$ gas injection on hot-spot temperature and core impurity concentrations in JET-ILW were presented in this paper. Besides the fueling rate, the impact of plasma current and separatrix density is discussed. This paper is structured as follows: section 2 presents impurity diagnostics description and analysis methods including a newly developed relative calibration technique for the JET VUV spectrometer, in presence of the ILW. A combination of measurements from the SXR, XUV and VUV diagnostics provide comprehensive information about tungsten and mid-Z impurities behaviour. The experimental results showing the impact of gas injection and sepatartrix density on hot-spot temperature and impurities are presented in section 3 . Tentative physics interpretation of the hot-spots formation due to fast ion losses is presented in section 4 and followed by drawing conclusions.

\section{Diagnostics and analysis methods}

\subsection{VUV/XUV spectroscopy diagnostics}

The JET VUV SPRED survey spectrometer [12-13] (known locally as KT2 diagnostic) was routinely used to give impurity data for the operation of the JET machine. The diagnostic has a horizontal line-of-sight (1-o-s) (illustrated in Figure 2 as red line) looking at the vessel mid-plane via a gold ( $\mathrm{Au}$ ) coated spherical mirror, allowing it to view the emission from the core and scrape-off layer of the plasma. Signal was detected by a micro-channel plate (MCP) and a phosphor screen from which light was coupled by a fiber optic cable to a 2048 pixels Photo Diode Array (PDA). The diagnostic was equipped with a $450 \mathrm{~g} / \mathrm{mm}$ holographic grating and measured spectra in the 100-1100 A wavelength range, with a spectral resolution of $5 \AA$. The shortest time resolution was $11 \mathrm{~ms}$, although $50 \mathrm{~ms}$ was routinely used. With the carbon plasma facing components (PFCs), the long wavelength of VUV spectrum was dominated by low-Z impurities like carbon $(C)$ and oxygen $(\mathrm{O})$, while the short wavelength range by different mid-Z impurities like nickel $(\mathrm{Ni})$, iron $(\mathrm{Fe})$, copper $(\mathrm{Cu})$, chromium (Cr), molybdenum (Mo) making this range particularly valuable from the point of view of diagnosing high temperature plasmas. After installation of the ITER-like wall (ILW) at JET, which consists of a full tungsten (W) divertor and beryllium (Be) main chamber PFCs, the spectrum also contains intense $\mathrm{W}$ features (see Figure 3 which shows recorded spectrum in JET Pulse No. (JPN) 92394). The
XUV/VUV spectrometer looking from the top of the machine towards the JET divertor, (as illustrated in figure 2) consists of three instruments, two SPRED spectrometers [13] (known locally as KT7/1 and KT7/2) and a Schwob-Fraenkel SOXMOS instrument [14] (known locally as KT7/3). The double SPRED used $450 \mathrm{~g} / \mathrm{mm}$ and $2105 \mathrm{~g} / \mathrm{mm}$ holographic grating, respectively. The higher resolution instrument observed spectra from $140 \AA$ to $443 \AA$ with a spectral resolution of $\sim 1 \AA$. The SOXMOS XUV spectrometer equipped with a $600 \mathrm{~g} / \mathrm{mm}$ grating, was set to record spectra in the wavelength range 40-70 $\AA$. The $165.4 \AA, 192.0 \AA$ and $153.5 \AA$ spectral lines were used to determine respectively $\mathrm{Ni}$, $\mathrm{Fe}$ and $\mathrm{Cu}$ concentrations in the plasma, using the method described in detail by Czarnecka et al. in Ref. [15]. This technique relied on the absolutely calibrated line intensities measured by the KT2 diagnostics, as well as simulations involving the universal transport code (UTC) [16]. To provide a quantitative measurement of the W-concentration, the XUV spectrometer, which observed a quasicontinuum of the $\mathrm{W}$-ions $\mathrm{W}^{27+}-\mathrm{W}^{35+}$, was used. This spectral feature was emitted at electron temperatures between $0.8-1.8 \mathrm{keV}$ [17]. W concentration was determined, as presented in [18], by taking into account intensity of measured quasicontinuum, proper atomic data for $\mathrm{W}$, the geometry, the electron temperature and density profiles. The VUV and XUV spectrometers provide intrinsic impurity concentrations, though the measurements were valid only in a limited radial range, where the ambient $T_{e}$ allows the ions emission (values of normalized radius r/a were presented in further figures). Furthermore, since measurements were line-integrated, the absolute concentrations inferred from these diagnostics depend on the poloidal distribution of the studied impurities.

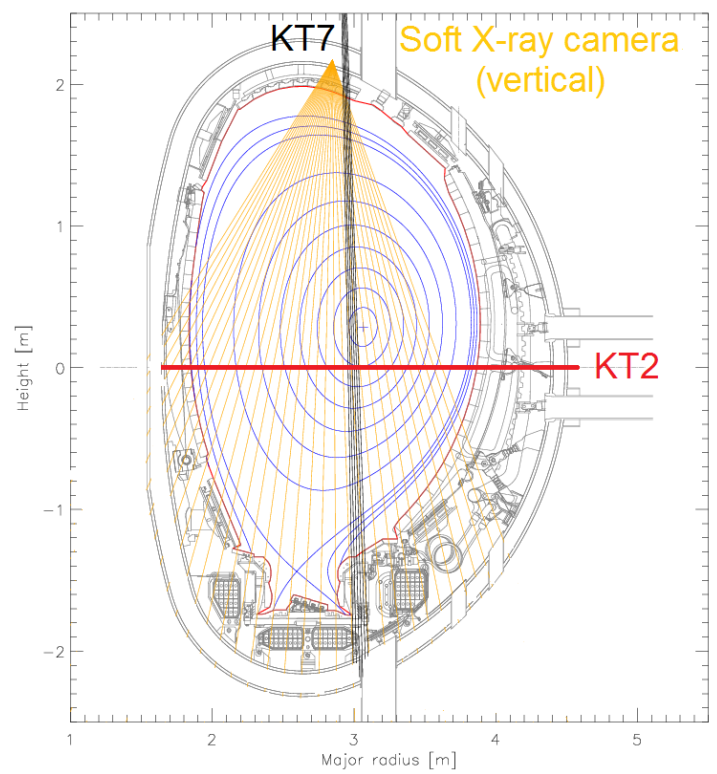

Figure 2. Lines of sight of JET VUV spectrometer KT2 (red horizontal line), XUV/VUV spectrometer KT7/1,2,3 (black vertical line) and SXR vertical (V) camera (orange lines) at JET tokamak. 


\subsubsection{New relative calibration method}

In order to fully exploit the line intensity measurements, sensitivity calibration of the spectrometers was necessary. In the

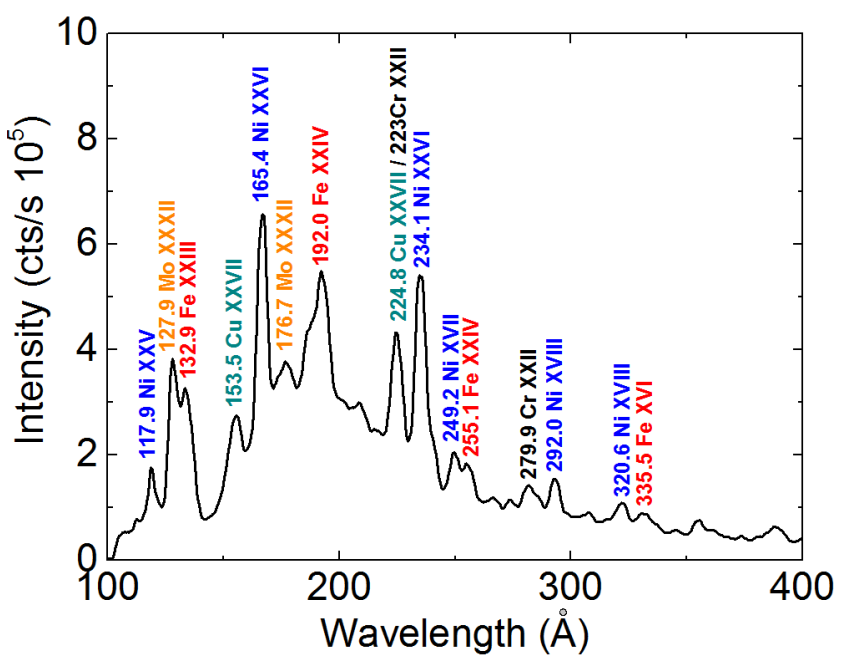

Figure 3. SPRED spectrum in the $100-400 \AA$ wavelength range for JET pulse No $92394 \mathrm{t}=8-8.5 \mathrm{~s}$.

case of visible instruments both relative and absolute sensitivity calibrations can be determined using a standard calibration lamp. However, the use of a local calibration source for VUV spectrometers was experimentally very difficult and the removal of vacuum instruments from the machine site to, for example, a synchrotron source was not possible due to beryllium contamination. As shown by Lawson et al. [19] it was possible to derive absolute sensitivity calibrations by combining two steps: using the branching ratio between the C IV $312.4 \AA$ VUV line and the lines at $5801 \AA$ and $5812 \AA$ from a calibrated visible spectrum to obtain the absolute calibration for one particular wavelength and use in situ method which involve the use of $\mathrm{Na}$ - and Li-like line intensity ratios of different elements to obtain a relative sensitivity curve and therefore calculate spectral response for other wavelengths as well. The reduction of the $\mathrm{C}$ level in the JET-ILW plasmas impacted on the difficulties with the branching ratio technique, because $\mathrm{C}$ lines were only occasionally observed, mostly in transient events such as a disruption. Relative intensities were temperature-dependent, which adds the uncertainty to the relative calibrations. This method was also suitable only when lines were well separated, which means that spectrometers with higher spectral resolution were more easily calibrated, (e.g. this method works much better for KT7/2 high resolution spectrometer than for KT2, where the relevant lines often overlap). Besides, it was found that an independent relative sensitivity calibration of KT7/2 instrument at JET was unchanged since last calibration reported in [20]. During the last campaigns the previously used method of obtaining relative intensity calibration [19] started to suggest a very strong deterioration of the sensitivity in the short-wavelength region of KT2 diagnostics, though the discrepancy could have been a result of some overlapping lines from different impurities. Therefore, to provide absolute mid-Z impurity concentrations a new relative calibration technique was proposed. It was based on cross-calibration with calibrated spectrometer using (simultaneous recordings of) the quasicontinuum of $\mathrm{W}$ in the relevant wavelength range (200-400 $\AA$ [19]). Analysis of a W ablation experiment [21] shows that around $100 \mathrm{~ms}$ after ablation, in sufficiently cold plasma (with maximum temperature not exceeding $2 \mathrm{keV}$ ), the same radiating cloud of W was viewed by all the VUV spectrometers with different 1o-s (e.g. horizontal and vertical). This radiation provides a possible tool for cross-calibration of the relative sensitivity of different VUV spectrometers, in the spectral regions where the $\mathrm{W}$ radiation was sufficiently strong. This was especially useful for the VUV diagnostic with low spectral resolution and possible line blending (like $\mathrm{Ni}, \mathrm{Cu}$ lines). In the case of too high core temperature, measured $\mathrm{W}$ spectra were too weak to have a proper signal to noise ratio and originated from relatively thin layer of plasma, which may not be symmetric and therefore different for horizontal and vertical l-o-s. As the radiation before and sufficiently after ablation was very similar, the difference of the spectra in studied temporal frames and the frames before ablation were considered as the "pure" ablated W spectrum. This spectrum was obtained by subtraction of two spectra, one averaged in the time from 200 to $100 \mathrm{~ms}$ before ablation, the second in the time 100 to 200 ms after ablation, to take into account the previously mentioned $100 \mathrm{~ms}$ time for the radiating $\mathrm{W}$ cloud to spread in the plasma. As the curve of relative sensitivity of the KT7/2 spectrometer could be considered correct, a curve of relative sensitivity of KT2 diagnostic were calculated from the ratio of KT2 to KT7/2 spectrum multiplied by the sensitivity of the KT7/2 diagnostic. Resulting curves obtained for different discharges were presented in figure 4 . Tungsten spectra after the new calibration were consistent with those from KT7/2 spectrometer. The calibration results show a large loss of detector sensitivity in the short-wavelength region in comparison to the calibration made for JET C-wall [15]. It can be also seen that the shapes of the relative calibration curves were pretty similar for different JET-ILW campaigns (carried out in 2015-2016), as the pulse numbers cover from 88500 to 92300. Resulting calibration was similar to the calibration curve obtained using the method developed by Lawson [19], calculated from the data from older ILW campaigns. The loss of sensitivity at short wavelengths was much less severe, if still present, than the calibrations from recent campaigns suggested (the sensitivity at $165 \AA$ calculated from line method was three times worse than from this calibration), and 
was thought to be due to a deterioration in the grating coating. Less likely explanations were degradation of the CuI coating on the front face of the microchannel plate (MCP) or an absorbing deposited film within the channels themselves. It was noted that throughout the period in which a loss of sensitivity has been observed, the second order spectrum, which was normally absent from SPRED spectra, increased in intensity. This can only be explained by a change in the grating properties, providing further evidence of a change in the grating itself.

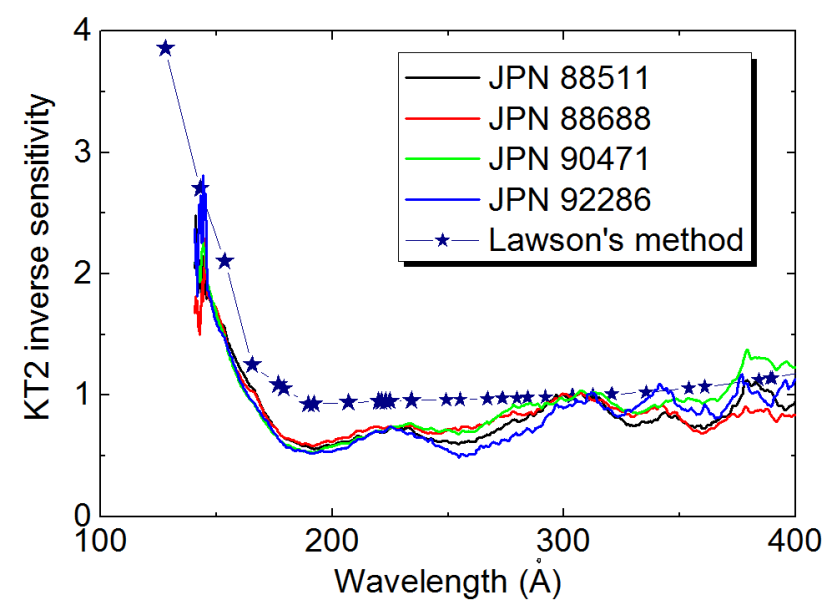

Figure 4. The relative inverse sensitivity calibration at short wavelengths for four pulses with W ablation experiments. Results were compared with calibration curves derived from $\mathrm{Na}$ and $\mathrm{Li}$ like doublet ratios $\left(\mathrm{S}^{-1}\right.$ at $\left.312.4 \AA=1\right)$ using $\mathrm{K}$. Lawson's method presented in [15].

\subsection{SXR diagnostic}

The SXR diagnostic [23] was essential for providing a quantitative estimate of high- $Z$ impurity content in the main plasma of JET. Since 2005 the SXR system consists of 3 cameras, two with vertical orientation $(\mathrm{V}, \mathrm{T})$ and one with horizontal $(\mathrm{H})$. The vertical cameras, due to their favourable viewing geometry, were often used for characterization of poloidal asymmetries. Due to a technical problem with camera $\mathrm{T}$, the presented results only make use of camera $\mathrm{V}$ with 35 lines-of-sight (1-o-s), presented in figure 2. By using camerra $\mathrm{V}$ it was possible to determine the profiles of impurity concentration and the 2D-tomographic reconstruction of $\mathrm{W}$ radiation in SXR range. The 1-O-S integrals were performed using their geometry, a magnetic equilibrium reconstruction from EFIT, and taking into account the 250 microns Be filter (with transmision from $1.5 \mathrm{keV}$ ). Quantitative diagnosis of the W content in this approach was described in detail in Ref. [18]. The SXR camera measurements were deconvolved using the assumption that all Bremsstrahlung was emitted due to low-Z impurities which does not exhibit poloidal asymmetries, while the additional radiation was attributed to high- $\mathrm{Z}$ and mid- $\mathrm{Z}$ impurities showing poloidal asymmetries mostly attributed to mass-dependent centrifugal effects. The low- $Z$ content was projected into a $\mathrm{Be}$ concentration obtained via the $Z_{\text {eff- }}$ measurement from visible Bremsstrahlung allowing to take also recombination radiation into account. The recombination radiation was generally not a major player in the SXR with respect to the Bremsstrahlung, as above $T_{e}=2 \mathrm{keV}$ the contribution to the SXR was less than $20 \%$. The uncertainty on separating the low- $Z$ from the high- $Z$ radiation becomed smaller for larger high- $Z$ content and as soon as the concentration of $\mathrm{W}$ was above $10^{-5}$ and the plasma temperature was above $2 \mathrm{keV}$ the separation was straightforward. However, it remains somewhat ambiguous which mid- $Z$ and high- $Z$ species in the plasma were responsible for the radiation in the soft $X$-ray range. The heavy impurity proportions cannot be disentangled quantitatively at the moment. This would require a quantitative, spectroscopic evaluation of all contributors with their radial distribution. Thus assuming all SXR contributions beyond Bremsstrahlung was emitted from $\mathrm{W}$ was one extreme yielding the highest bolometric implications from the SXR radiator. The physical meaning of the results may be considered preliminary, however, future corrections of the data will not change the main thrust of this paper as most of the conclusions rely on large changes of the soft x-ray emissions, outside of the uncertainties.

\section{Experimental Results}

All examples discussed here were hybrid discharges with magnetic field $\mathrm{B}_{\mathrm{o}}=2.76 \mathrm{~T}$, plasma current between $\mathrm{I}_{\mathrm{p}}=2.0$ - 2.4, $\mathrm{P}_{\mathrm{NBI}}=25 \mathrm{MW}$, and $\mathrm{P}_{\mathrm{ICRH}} \geq 5 \mathrm{MW}$ in dipole phasing using simultaneously first harmonic hydrogen $(\mathrm{N}=1 \mathrm{H})$ minority $\sim 2$ $\%$ and $\mathrm{N}=2 \mathrm{D}$ majority. In these conditions, the plasmas were characterized by low collisionality and high $\mathrm{q}_{95}$. Time evolution of the selected parameters for three discharges with constant $\mathrm{I}_{\mathrm{p}}=2.2 \mathrm{MA}$ and different $\mathrm{D}_{2}$ gas injection rate from the mid-plane gas injection module (GIM6), affecting hot spot temperature in the poloidal limiter NPL3B, were presented in figure 5. Previously obtained experimental results showed that distributed mid-plane gas injection was the best recipe for overall RF coupling improvement at gas levels compatible with high performance scenario development $[6,24,25]$. There were indications that this type of distributed gas injection also has a beneficial impact on RF-induced impurity content but the physics mechanisms behind such observation were still under study [24]. The gas flow from GIM6 was varied in the pulses illustrated in figure 5, but the gas flow from other valves was adjusted so that the total gas flow only varied by $\sim 17 \%$. It is not believed that changes in gas injection location in the main chamber have a significant impact on the plasma performance, either through changes in ELM frequency or plasma density, which affects neutral beam penetration. However, low gas injection rates are used in these 
experiments to allow a high $\mathrm{H}$-mode pedestal temperature and, if higher total gas injection rates were needed to mitigate main chamber hot spots, it is expected that this would affect the plasma fusion performance. The hot-spot temperature was measured by the real-time wall protection camera.

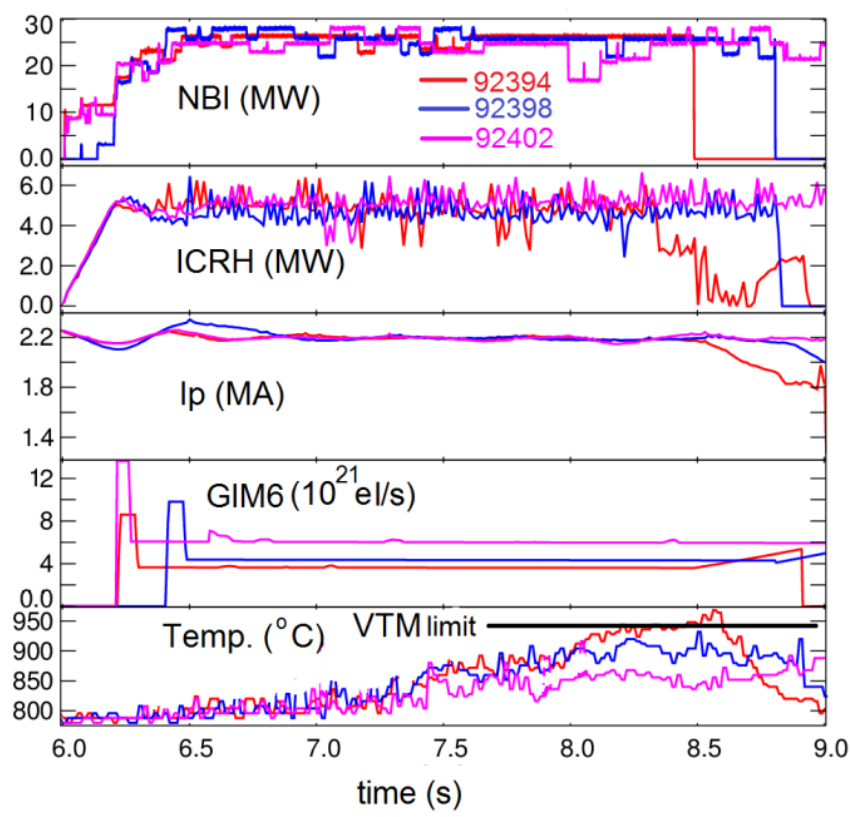

Figure 5. Time evolution of NBI and ICRH heating power, for pulses with the same plasma current $\mathrm{Ip}=2.2 \mathrm{MA}$, with different $\mathrm{D}_{2}$ gas injection from the mid-plane gas injection module GIM6 and hot spot temperature in the poloidal limiter NPL3B. VTM limit was presented as a black horizontal line.

The largest temperature $\left(\mathrm{T}_{\max }=940^{\circ} \mathrm{C}\right)$ was observed at lowest gas rate in pulse 92394 which therefore was stopped by the real-time wall protection system [24] (vessel thermal map (VTM) limit presented in Figure 5). Unfortunately heat-load deconvolution cannot be performed on the temperature data shown in Figure 5 because the dynamic range of the protection camera used was too narrow $\left(\mathrm{T}>750{ }^{\circ} \mathrm{C}\right)$. The Infra-Red Scientific camera, which did measured temperatures starting from $200 \mathrm{deg}$. C did not see the narrow poloidal limiter (NPL3B) where the hotspot was observed. The maximum temperature reached in similar discharges with varying $I_{p}$ were presented in Figure 6 as function of the $\mathrm{D}_{2}$ gas injection rate. Dedicated investigations showed the hot-spot temperature decreasing with local $\mathrm{D}_{2}$ gas injection (from GIM6), allowing the pulses to run with acceptable temperature values on protruding vessel components. In addition, at fixed gas injection rate, higher plasma current discharges (characterized by higher separatrix density) were less prone to hot-spot formation. As presented in Figure 7, a decrease of the $\mathrm{W}$ and mid-Z impurity concentration with $\mathrm{D}_{2}$ gas injection rate was equally observed. $\mathrm{Ni}$ and $\mathrm{Fe}$ impurities come from Inconel or similar Ni based alloys components at the outer main chamber wall. W impurities comes mainly from $\mathrm{W}$ divertor and associated divertor baffles but can also be sputtered from plasma facing components located in the main chamber (such as the NBI shine-through protection plates). However, it must be noted that mid- $\mathrm{Z}$ and high- $\mathrm{Z}$ impurities migrate throughout the vessel and were deposited in the divertor and main chamber limiters. As it can be seen in Figure 7f, the $\mathrm{Cu}$

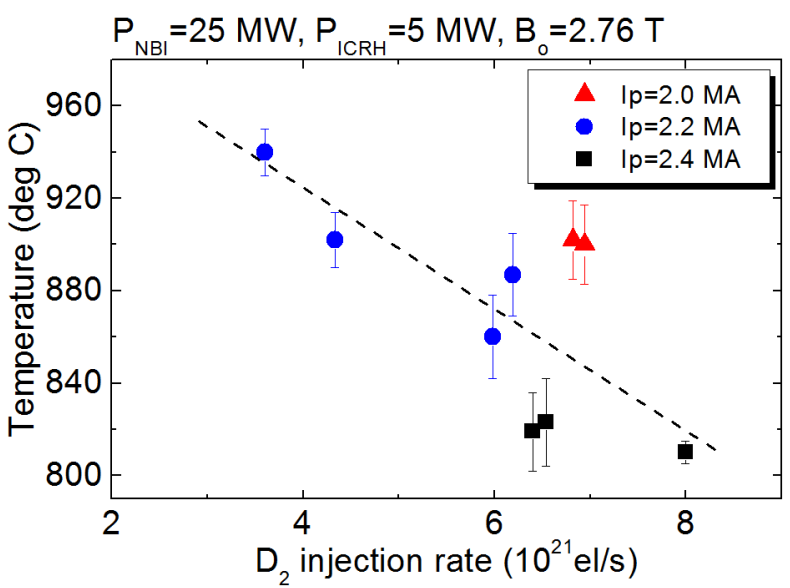

Figure 6. Hot-spot temperature on the narrow poloidal limiter NPL3B determined form the protection camera signals as a function of $\mathrm{D}_{2}$ gas injection rate from GIM6. Note: Linear fits were calculated for group of points with $\mathrm{I}_{\mathrm{p}}=2.2 \mathrm{MA}$

concentrations remains unchanged in this series of discharges. The presence of $\mathrm{Cu}$ in JET-ILW high power plasmas was associated with the use of the NBI heating system. The duct liners consist of a number of actively cooled hypervapotrons made of copper. They act as a sputtering source since they are intercepting high energy particles. The acceleration grids, residual ion dumps, scrapers, protection plates also contain copper and this can all contribute to the copper source coming from the beamline. The error bars presented in Figure 7 were obtained by propagating an uncertainty of the measured line intensity, electron densities and temperatures. The systematic uncertainties from errors in the poloidal fitting, unaccounted up-down asymmetries and geometry on the $\mathrm{W}$-densities were small as compared to those from atomic data. The same decreasing trend was observed in the behaviour of the $\mathrm{Z}_{\mathrm{eff}}$ determined from visible spectroscopy diagnostic (see Figure 8a). Calculations of the contribution of mid-Z impurities to effective charge $\left(\Delta Z_{\text {eff }}\right)$, by the use of data from the VUV spectroscopy and the $\Delta Z_{\text {eff }}$ equation from Ref. [15], were presented in Figure $8 \mathrm{c}-\mathrm{e}$ ). The r/a values were determined by calculation of the maximum emissivity of the $\mathrm{Ni}, \mathrm{Fe}$ and $\mathrm{Cu}$ Li-like ions along the VUV 1-o-s. Emissivity was dependent on the photon emissivity coefficient (pec), the electron temperature, electron density and impurity density. It was found that highest $\Delta \mathrm{Z}_{\mathrm{eff}}<0.6$ came from $\mathrm{Ni}$. Due to much lower concentration in comparison to $\mathrm{Ni}$, and despite the higher Z, W contribution to $\mathrm{Z}_{\mathrm{eff}}$ (see Fig. 8b) was lower than from Ni. As it can be seen in Figure 9, the total radiated power, measured by bolometry diagnostic [26], slightly decreased 


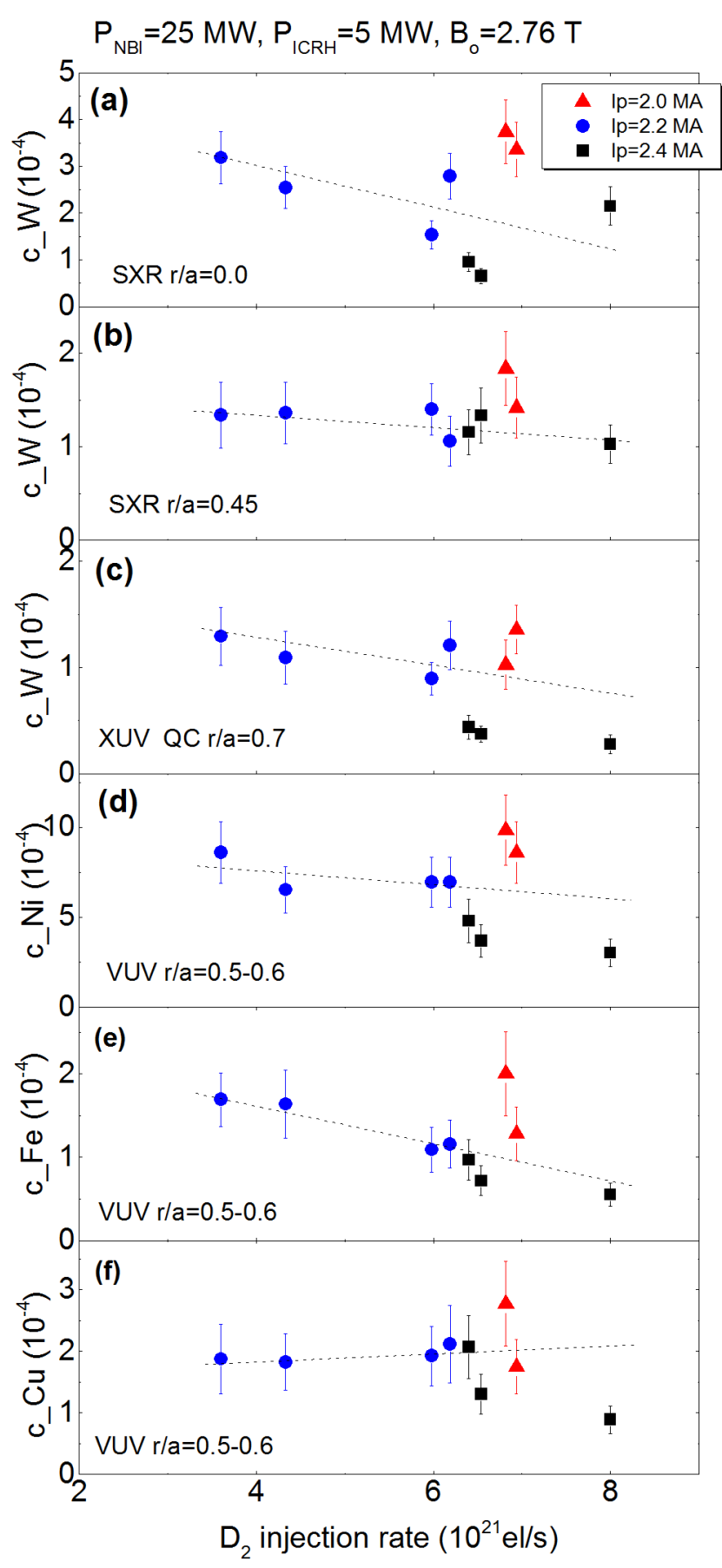

Figure 7. Concentration of a) $\mathrm{W}$ at $\mathrm{r} / \mathrm{a}=0.0$, b) $\mathrm{W}$ at $\mathrm{r} / \mathrm{a}=0.45$ derived from the SXR diagnostic, c) $\mathrm{W}$ at $\mathrm{r} / \mathrm{a}=0.7$ derived from the XUV diagnostic by the use of methods described in [18] and d) $\mathrm{Ni}$ at $\mathrm{r} / \mathrm{a}=0.5-0.6, \mathrm{e}) \mathrm{Fe}$ at $\mathrm{r} / \mathrm{a}=0.5-0.6, \mathrm{f}) \mathrm{Cu}$ at $\mathrm{r} / \mathrm{a}=0.5-0.6$ derived from the VUV diagnostic using method described in [15]), as a function of $\mathrm{D}_{2}$ injection rate for discharges with different plasma current $\mathrm{I}_{\mathrm{p}}$. Note: Linear fits were calculated for the group of points with $\mathrm{I}_{\mathrm{p}}=2.2 \mathrm{MA}$.

with $\mathrm{D}_{2}$ gas injection rate. Roughly half of the auxiliary power was radiated away from the plasmas. $\mathrm{W}, \mathrm{Ni}, \mathrm{Fe}$ and $\mathrm{Cu}$ radiated power, was estimated based on impurity density measurements from XUV and VUV data and the appropriate cooling rates. It was found that $\mathrm{W}$ was the main intrinsic radiator. However, the W radiated power shown in Figure 9, which was calculated from the $\mathrm{W}$ quasicontinuum may be somewhat underestimated. It is due to due to observed poloidal asymmetries and a limitation of the impurity density calculations which were only well constrained at a certain r/a. A fraction of the missed total radiated power can be also related to the $\mathrm{W}$ radiation not detected by the XUV diagnostic. A small part of the radiation can be associated with other metallic impurities not taken into account (e.g. Mo). Assuming all SXR contributions above Bremsstrahlung was emitted from $\mathrm{W}$, delivers the upper bound of the radiated power. Obtained results shows that, the mid- $Z$ impurities give rise both to $Z_{\text {eff }}$ and to radiated power. Thus, also affect the SXR radiation.

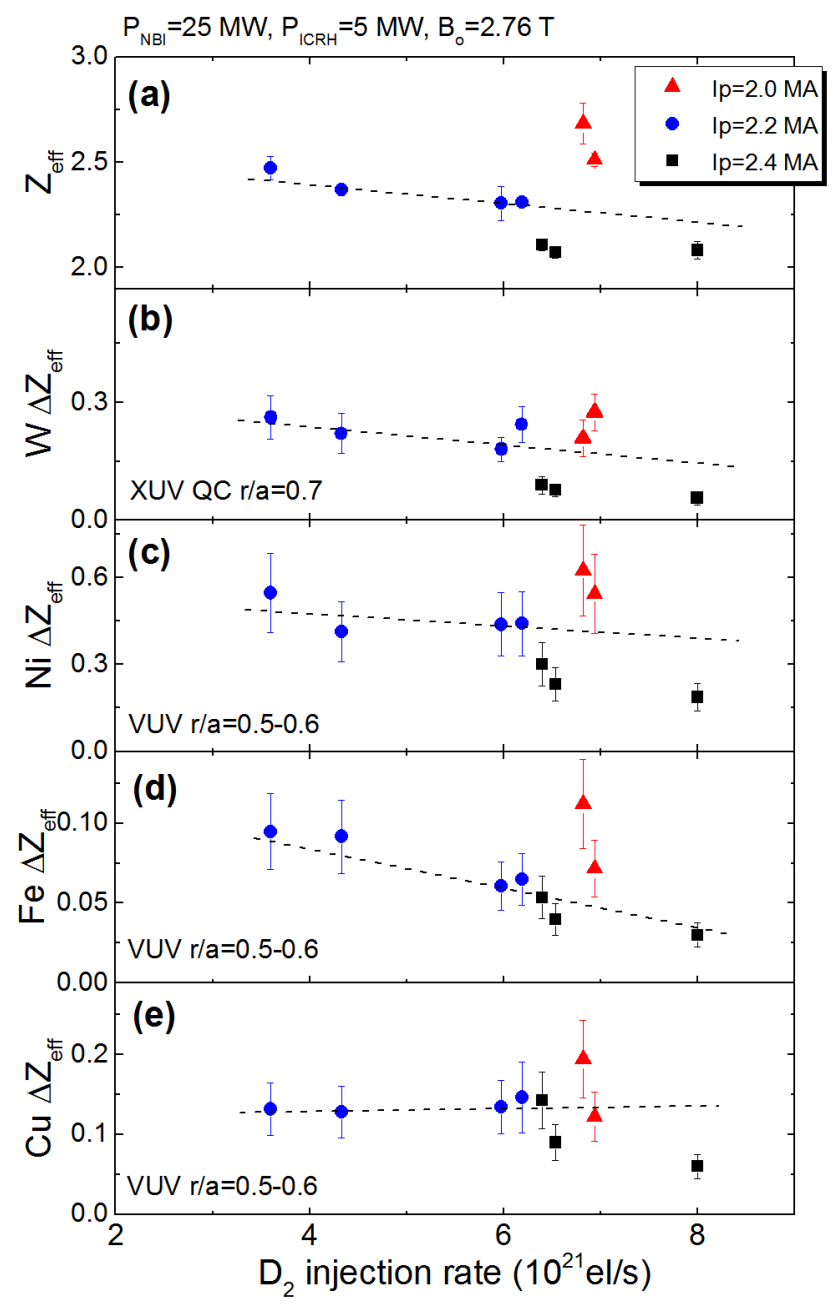

Figure 8. a) $\mathrm{Z}_{\mathrm{eff}}$ determined form visible spectroscopy diagnostic and contribution of b) $\mathrm{W}$ to $Z_{\text {eff }}$ derived from the XUV diagnostic, c) $\mathrm{Ni}$, d) $\mathrm{Fe}$ and e) $\mathrm{Cu}$ determined based on data from the VUV spectroscopy diagnostic [15], as a function of $\mathrm{D}_{2}$ injection rate for different $\mathrm{I}_{\mathrm{p}}$. Note: Linear fits were calculated for the group of points with $\mathrm{I}_{\mathrm{p}}=2.2 \mathrm{MA}$. 
Different trends of the hot spot temperature, impurity concentration and $Z_{\text {eff }}$ were observed when plasma current $\left(I_{p}\right)$ was changed. Changes in the $I_{p}$ have a strong impact on the plasma-wall interaction, via modifications in the edge density. Figure 10 shows hot spot temperature in plasmas with different $I_{p}$ at the same $D_{2}$ gas injection rate $\left(\sim 6-7 \times 10^{21} \mathrm{el} / \mathrm{s}\right)$ as a function of the separatrix density $\left(\mathrm{n}_{\mathrm{sep}}\right)$. It was observed that at approximately constant gas injection rate, both the hotspot temperature and the core metallic impurity content (presented in Figure 11) decrease with the $\mathrm{n}_{\text {sep }}$. In Figure 12

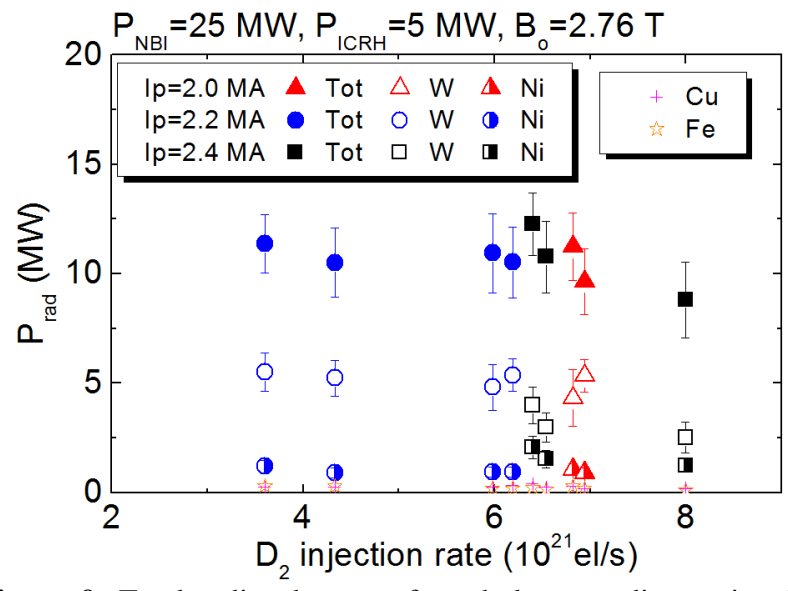

Figure 9. Total radiated power from bolometry diagnostics (full symbols) with $\mathrm{W}$ radiated power (open symbols) estimated from XUV data (at $\mathrm{r} / \mathrm{a}=0.7$ ), $\mathrm{Ni}$ radiated power (half open symbols), $\mathrm{Fe}$ radiated power (stars), and $\mathrm{Cu}$ (crosses) radiated power estimated based on VUV data (at $\mathrm{r} / \mathrm{a}=0.5-0.6$ ) as a function of $\mathrm{D}_{2}$ injection rate for discharges with different $I_{p}$.

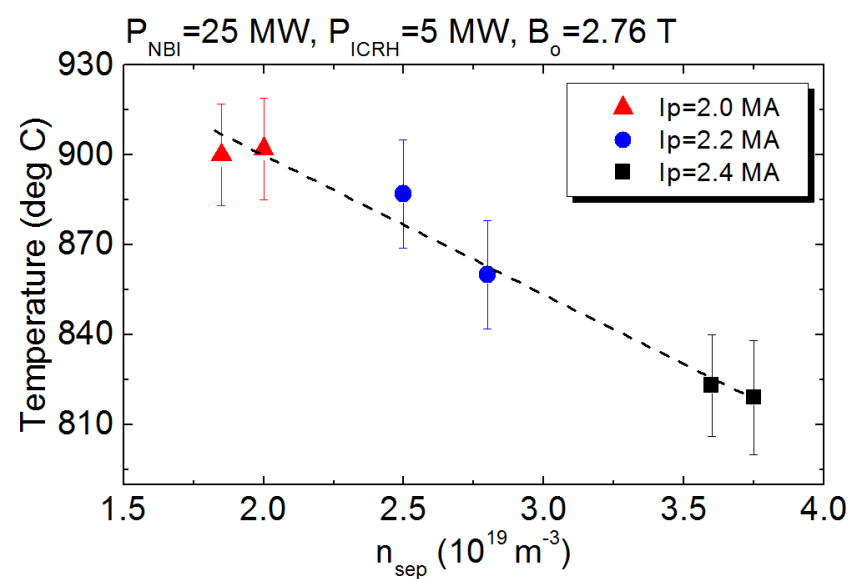

Figure 10. Hot-spot temperature (averaged $0.5 \mathrm{~s} \sim \mathrm{t}=8 \mathrm{~s}$ ) on the narrow poloidal limiter NPL3B determined from the protection camera signals as a function of separatrix density for different plasma current $I_{p}$.

the 2D SXR radiation pattern (mainly attributed to $\mathrm{W}$ in these pulses) was shown for 3 discharges with fixed input power $\left(\mathrm{P}_{\mathrm{NBI}}=25 \mathrm{MW}, \mathrm{P}_{\mathrm{ICRH}}=5 \mathrm{MW}\right)$ and different plasma current (and $n_{\text {sep }}$ ) whose time traces were presented in Figure 13. One readily sees that the core $\mathrm{W}$ radiation was reduced at higher plasma current and that the poloidal extent of the off-axis radiation region ('mantle') was increased. Poloidal asymmetry within flux surfaces was caused by the centrifugal force on W. Off-axis peaks occur when the radial transport across flux surfaces has a sign inversion near the pedestal top (outward inside the pedestal, inward in the pedestal) [8-10]. This was common in JET shots before the main ion density became too peaked and was due to the main ion density gradients which determine the direction of neoclassical convection. When main ion density become more peaked, the convection changed sign and it drove inward $\mathrm{W}$ transport and then accumulation on-
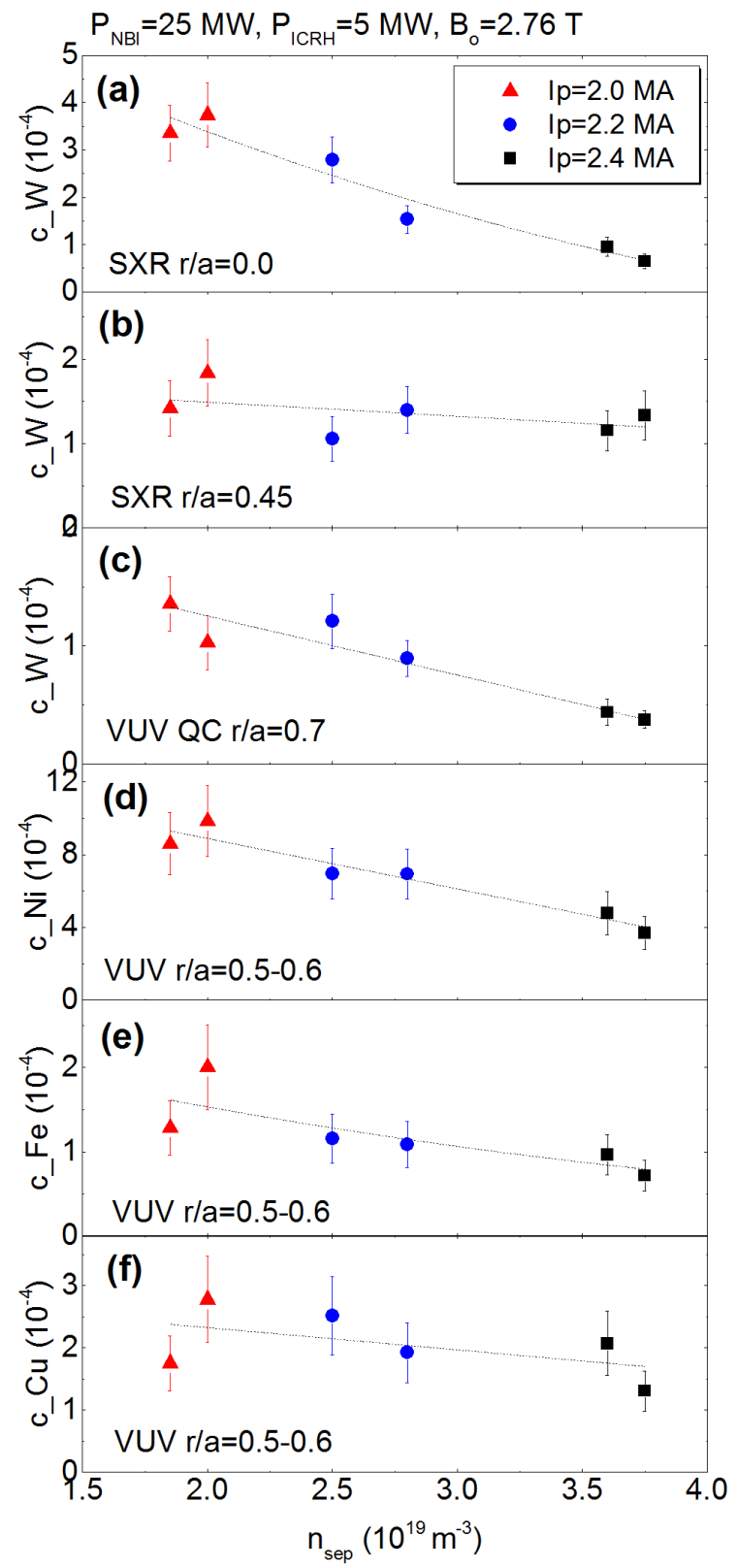

Figure 11. Impurity concentration as a function of separatrix density for different plasma current $\mathrm{I}_{\mathrm{p}}$ at the same $\mathrm{D}_{2}$ gas injection rate ( 6- 
$7 \times 10^{21} \mathrm{el} / \mathrm{s}$ ). Concentration of a) $\mathrm{W}$ at $\mathrm{r} / \mathrm{a}=0.0$, b) $\mathrm{W}$ at $\mathrm{r} / \mathrm{a}=0.45$ derived from the SXR diagnostic, c) $\mathrm{W}$ at $\mathrm{r} / \mathrm{a}=0.7$ derived from the XUV diagnostic by the use of methods described in [18] and d) $\mathrm{Ni}$ at $\mathrm{r} / \mathrm{a}=0.5-0.6, \mathrm{e}) \mathrm{Fe}$ at $\mathrm{r} / \mathrm{a}=0.5-0.6, \mathrm{f}) \mathrm{Cu}$ at $\mathrm{r} / \mathrm{a}=0.5-0.6$ derived from the VUV diagnostic using method described in [15]).

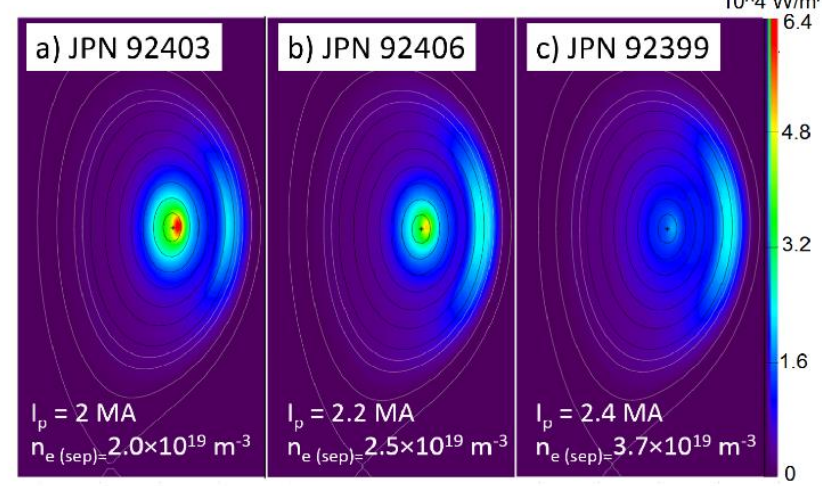

Figure 12. 2D-profiles of SXR-radiation distributed over the JET poloidal cross-section (averaged $0.4 \mathrm{~s} \sim \mathrm{t}=8$ ) $\mathrm{s}$ for a) JPN 92403 with $\left.\mathrm{I}_{\mathrm{p}}=2 \mathrm{MA}, \mathrm{n}_{\text {sep }}=2 \times 10^{19} \mathrm{~m}^{-3}, \mathrm{~b}\right) \mathrm{JPN} 92406$ with $\mathrm{I}_{\mathrm{p}}=2.2 \mathrm{MA}, \mathrm{n}_{\text {sep }}$ $=2.5 \times 10^{19} \mathrm{~m}^{-3}$, and $\mathrm{c}$ ) JPN 92399 with $\mathrm{I}_{\mathrm{p}}=2.4 \mathrm{MA}, \mathrm{n}_{\text {sep }}=3.75 \times 10^{19}$ $\mathrm{m}^{-3}$.

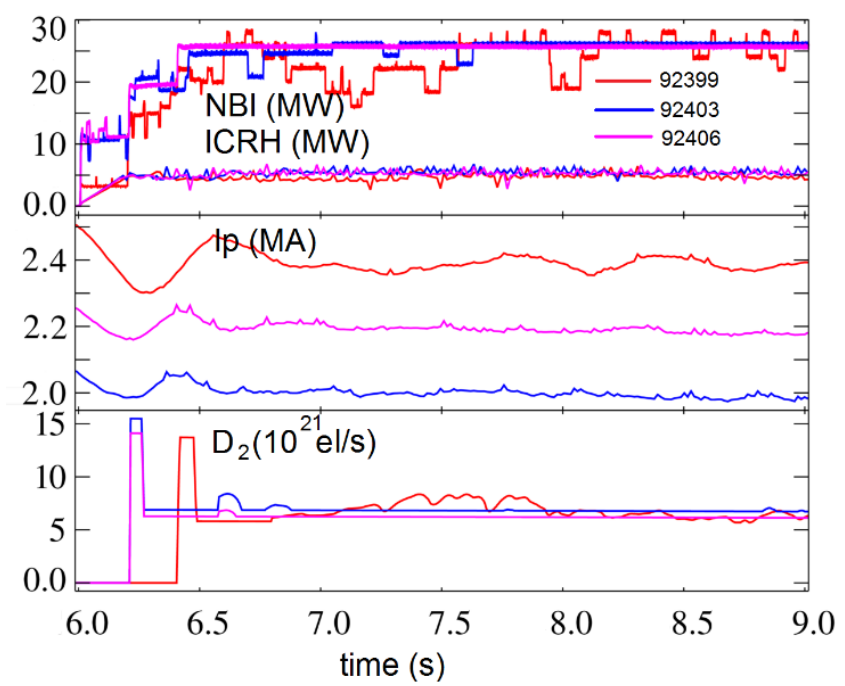

Figure 13. Time evolution of NBI and ICRH heating power, plasma current and $\mathrm{D}_{2}$ gas injection rate for the selected discharges with different $I_{p}$ and the same gas injection rate.

axis was observed. In presented cases, the convection reversed direction more than once across the profile. As can be seen in Figure 14, the rotation velocity evaluated from the observed poloidal in-out asymmetry remains the same for the three analysed pulses. Consistency checks of the results with other independent diagnostics, e.g. provided by charge exchange recombination spectroscopy (CXRS), are necessary as an indicator of uncertainties between the two diagnostics. Unfortunately, CXRS data were not available for the analysed discharges. However, consistency checks were performed with the rotation velocity obtained from the high resolution $\mathrm{x}$ - ray crystal spectrometer (XCS) (see Figure 14) that observed the spectrum around the resonance line of the helium-like $\mathrm{Ni}$ ion. Since the velocity derived from assuming $\mathrm{W}$ radiation dominated the SXR was smaller than the measured one from XCS then the contributed impurities has a smaller mass than W. Such observation supports the results showing impact of mid-Z impurity content in the analysed discharges. Calculated rotation exceeds the XCS data when $\mathrm{Ni}$ as the main SXR radiator was assumed. It should be noted that ions with significantly different mass can in some circumstances have different velocities. It should be also noted that poloidal asymmetries, of the $\mathrm{W}$ spatial distribution might lead to an underestimation of the W-concentration determined from the VUV diagnostic, however, spectroscopy provides a direct method to determine the $\mathrm{W}$ concentration independently of other radiators in the plasma.

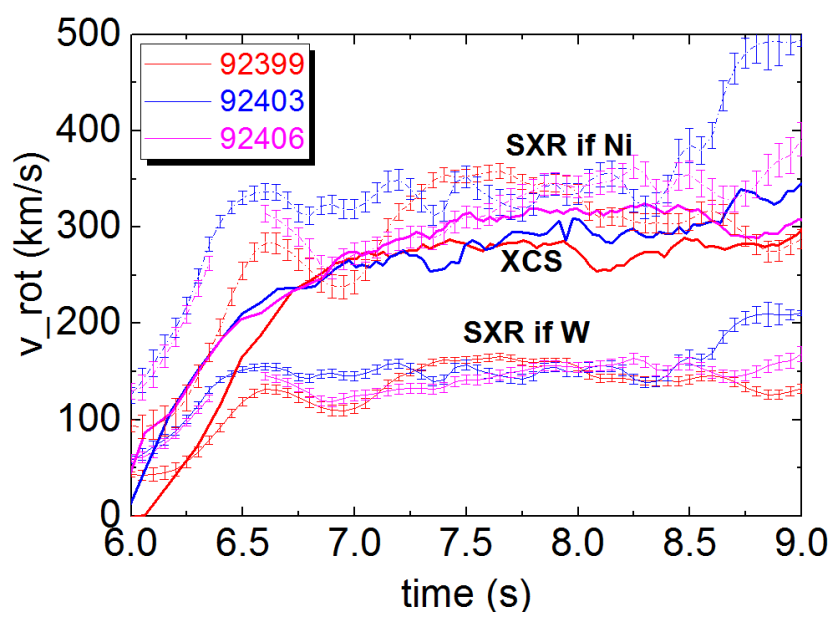

Figure 14. Time evolution of the rotation velocity at $\mathrm{r} / \mathrm{a}=0.45$ obtained from the SXR data when $\mathrm{W}$ or Ni (dash dot lines) was assumed as the main SXR radiator, and at r/a 0.4 obtained from XCS diagnostic (solid lines).

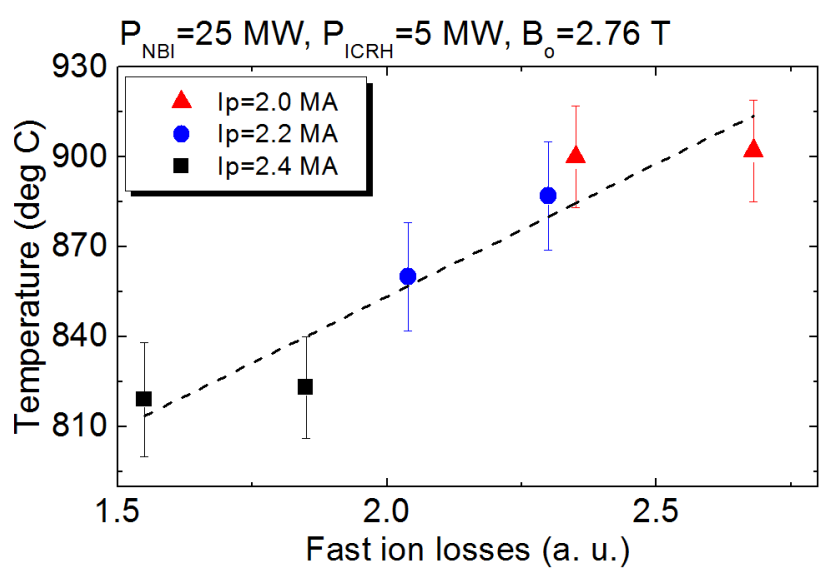

Figure 15. Hot-spot temperature (averaged $0.5 \mathrm{~s} \sim \mathrm{t}=8 \mathrm{~s}$ ) on the narrow poloidal limiter NPL3B determined from the protection 
camera signals as a function of fast ion losses determined from the KA3 fast particle diagnostic.

\section{Mechanism for the formation of the hot-spots - discussion}

The mechanism responsible for the formation of the hot-spots it seems to be linked to fast ion losses. Plasma current and the separatrix density were linked to each other and have a strong impact on the plasma-wall interaction, via changes in the fastion losses and orbit topology. Such effects cannot be disentangled in the experiment. As it can be seen in Figure 15, at constant gas rate, hot-spot temperature increased with fastion losses, obtained from the KA3 fast particle diagnostic [27]. However, KA3 data do not show a typical ICRH accelerated footprint for D above $200 \mathrm{keV}$. On the other hand, it has to be noted that only a very small sub-population of D (or D-beam) ions were accelerated to high energies with $\mathrm{N}=2$ ICRH acceleration but they may carry a substantial amount of the RF wave energy. Since there were few particles only but with high energy, to quantify this, full-wave modelling with orbit effects coupled to a 3D Fokker-Planck solver is required which was outside the scope of this paper. Additional studies, presented in [26], shows that there were strong bursts of fusion product ion losses during fishbone instabilities. The detected losses were associated with the highly energetic D-D fusion products ( $3 \mathrm{MeV}$ protons and $1 \mathrm{MeV}$ tritons).

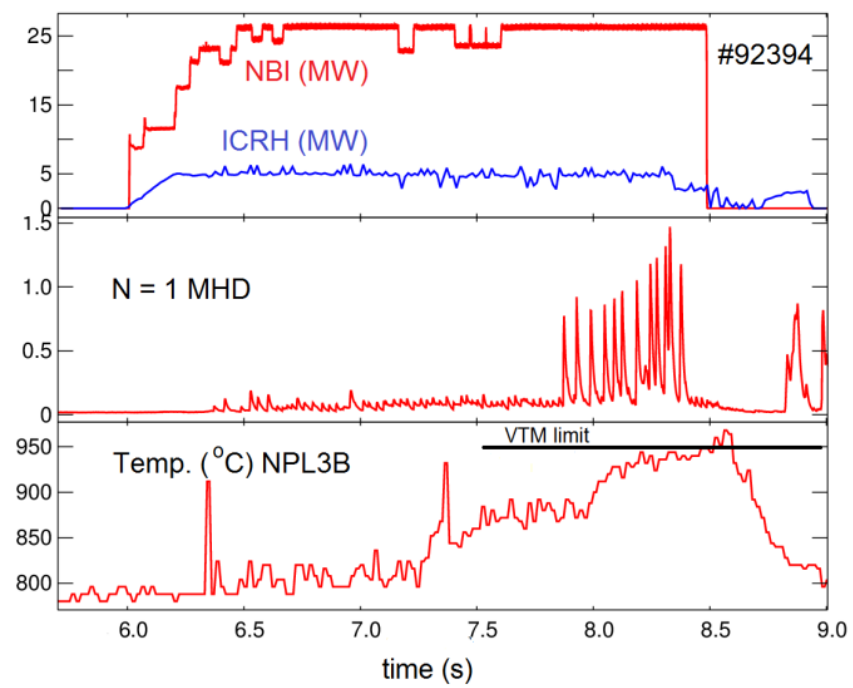

Figure 16. Time evolutions of NBI and ICRH heating power, $\mathrm{N}=1$ MHD instabilities and hot-spot temperature on the narrow poloidal limiter NPL3B for the JPN 92394 with $\mathrm{I}_{\mathrm{p}}=2.2 \mathrm{MA}$, GIM6 $=3.6 \times 10^{21}$ $\mathrm{e} / \mathrm{s}, \mathrm{n}_{\text {sep }}=2.6 \times 10^{19} \mathrm{~m}^{-3}$.

Full orbit analysis presented in [28] indicated that the $\mathrm{MeV}$ range ions were likely to hit the wall near the outer mid-plane, that was, close to where the hot-spot on NPL3B was observed and that up to $25 \%$ of the fast fusion product ions can be lost during fishbones. However, the total power attributed to fusion products in these pulses was too low $(\mathrm{P}<=20 \mathrm{~kW})$ to explain the temperature increase observed in the poloidal limiters. Furthermore, although there were pulses for which the hot-spot temperature seems to increase after large fishbones become present (see Figure 16 showing the example of the discharge in which temperature limit was reached causing plasma stop by the protection system) there were other examples with considerable hot-spot temperatures which do not respond to the fishbone's onset (see Figure 17). Note that in D-T plasmas, where the fusion power will be significant, the fishbone losses may have a much larger impact on the plasma-wall interaction.

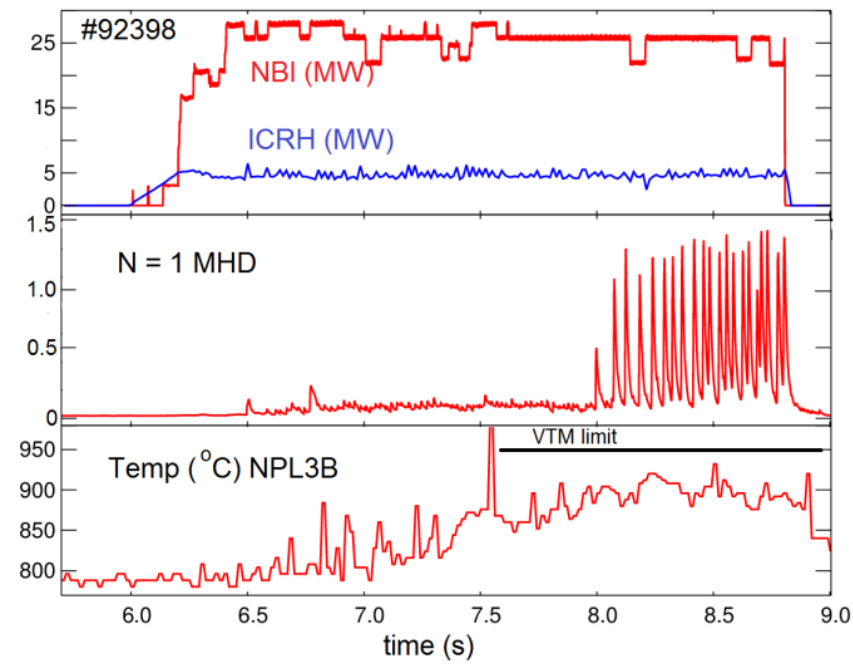

Figure 17. Time evolutions of NBI and ICRH heating power, $\mathrm{N}=1$ MHD instabilities and hot-spot temperature on the narrow poloidal limiter NPL3B for the JPN 92398 with $\mathrm{I}_{\mathrm{p}}=2.2 \mathrm{MA}$, GIM6 $=4.3 \times 10^{21}$ $\mathrm{e} / \mathrm{s}, \mathrm{n}_{\mathrm{sep}}=1.8 \times 10^{19} \mathrm{~m}^{-3}$.

\section{Conclusions}

In some high power hybrid plasmas, hot-spots on the outboard poloidal limiters were observed. The heat-loads could be minimized by local gas injection (keeping the total fueling constant) without affecting the core plasma properties, which would suggest that a scrape-of-layer mechanism (e.g. RF sheath effects) was responsible for the enhanced heat-loads observed. It was found that, the changes in gas injection level affect both hot-spots temperature and metallic impurity concentration. In the performed analysis, W profiles and poloidal asymmetries were obtained from the SXR diagnostic data, while the content of mid-Z impurities $(\mathrm{Ni}, \mathrm{Fe}, \mathrm{Cu})$ was assessed by means of VUV spectra. For proper quantitative estimation of the mid- $Z$ impurity concentration, the new relative calibration technique was shown to be a useful method in the ILW environment at JET. The calibration results showed loss of detector sensitivity in the short-wavelength region in comparison to the calibration made for JET C-wall. Reduction of hot-spot temperature and metallic impurities content with total $\mathrm{D}_{2}$ gas injection rate was observed. There was a clear correlation of these quantities with $I_{p}$ and the separatrix density. For lower $I_{p}$ (lower $n_{\text {sep }}$ ), hot-spot temperatures and impurity content was larger than at higher $I_{p}$ 
with similar fueling rate. Furthermore, there was also a correlation between the fast particle losses and the heat loads observed in the specific outer wall Be limiters. These observations would suggest that the fast ions impinging in the poloidal limiters come directly from the plasma rather than being accelerated in the scrape-off layer. But this latter observation contradicts the fact that local gas injection did not affect the core profiles, but nevertheless hot spots and impurities do change. One possible explanation was that the fast particle beam that hits a specific region of the limiter was somewhat scattered by the higher SOL density and neutral pressure and this in turn causes the fast ions to had a larger probability of colliding with an ions or neutrals before hitting the wall. This hypothesis has still to be confirmed. Prompt fusion product losses during large fishbones could also contribute to the enhanced plasma-wall interaction observed but the power produced by these reactions was on its own too low to explain the temperature rise observed in the outboard poloidal limiters. In summary, the actual mechanisms caused the heat loads - core losses or scrape-off-layer effects, were not yet understood. Further experimental investigations are planned and the numerical assessment to identify the process involved in mitigating the formation of hot-spots in future high power hybrid discharges is still ongoing.

\section{Acknowledgements}

This work has been carried out within the framework of the EUROfusion Consortium and has received funding from the Euratom research and training programme 2014-2018 and 2019-2020 under grant agreement No 633053. The views and opinions expressed herein do not necessarily reflect those of the European Commission. This scientific work was partly supported by Polish Ministry of Science and Higher Education within the framework of the scientific financial resources in the years 2014-2019 allocated for the realization of the international co-financed project..

\section{ORCID iDs}

A Czarnecka https://orcid.org/0000-0003-4931-728X

\section{References}

[1] H. Weisen et al. 2014 AIP Conference Proceedings 161277

[2] L. Horton et al. 2016 Fusion Engineering and Design 109_ 111925

[3] Chalis C et al 2015 Nucl. Fusion 55053031

[4] Mantsinen M J et al. 2017 European Physical Journal Web of Conferences 1573032

[5] Goniche M et al. (2017) Plasma Physics and Controlled Fusion 59055001

[6] Lerche E et al. 2016 Nuclear Fusion 56036022

[7] Klepper C C 2013 Journal of Nuclear Materials 438 S594

[8] Angioni C et al., 2014 Nuclear Fusion 54083028

[9] Casson F et al. 2015 Plasma Physics and Controlled Fusion 57 014031

[10] Angioni C et al. 2015 Physics of Plasmas 22055902
[11] Van Eester D et al., Proc. 26rd IAEA Fusion Energy, Kyoto, Japan, 17-22 October 2016, IAEA-CN-234, EX/P6-10.

[12] Coffey I H, R. Barnsley R 2004 Rev Sci Instrum 753737

[13] Fonck R J , Ramsey A T, Yelle R V 1982 Appl Optics 21 2115

[14] Schwob J L et al 1987 Rev. Sci. Instrum. 581601

[15] Czarnecka A et al. 2011 Plasma Phys. Control. Fusion 53 035009

[16] Whiteford A D et al. 2004 Proc. 31 ${ }^{\text {st }}$ EPS Conf., London, UK $28^{\text {th }}$ June $-2^{\text {nd }}$ July, vol. $28 \mathrm{G}$ (ECA) P-1.159

[17] Pütterich T et al 2008 Plasma Phys. and Control. Fusion 50085016

[18] Putterich T et al. 2013 Plasma Phys. Control. Fusion 55 124036

[19] Lawson K D, Coffey I H, Zacks J, Stamp M F 2009 J. Instrum. 4 P04013

[20] Lawson K D et al. 2011 Plasma Phys. Control. Fusion 53 015002

[21] Pawelec E et al. $44^{\text {rd }}$ EPS Conference on Plasma Physics, Vol. 41F, P5.157 (2017)

http://ocs.ciemat.es/EPS2017PAP/pdf/P5.157.pdf.

[22] Alper B et al. 1997 Review of Scientific Instruments 68778

[23] Lerche E et al 2015 Journal of Nuclear Materials 463634

[24] Jacquet P et al 2016 Nucl. Fusion 56046001

[25] Valcárcel D.F. et al. 2014 Fusion Engineering and Design 89243

[26] Huber A et al 2007 Fusion Engineering and Design 82 1327

[27] Kiptily V G et al. 2009 Nucl. Fusion 49065030

[28] Fitzgerald M et al. 2019 Nucl. Fusion 59016004 